\title{
QUANTUM-CHEMICAL QUASI-DOCKING
}

\author{
V.Sulimov $^{1,2}$, D. Kutov ${ }^{1,2}$, I. Ilin ${ }^{1,2}$, A. Sulimov ${ }^{1,2}$ \\ ${ }^{1}$ Dimonta Ltd., 117186, Russian Federation, Moscow, Nagornaya str., 15, bldg 8. \\ ${ }^{2}$ Research Computer Center of Lomonosov Moscow State University, \\ 119991, Russian Federation, Moscow, Leninskie Gory, 1, bldg 4.
}

DOI: 10.19163/MedChemRussia2021-2021-172

E-mail:vladimir.sulimov@gmail.com

Molecular dynamics and docking are the most important methods used for the search and design new inhibitors of a given target proteins responsible for disease progressing. Molecular dynamics is considered to be the most accurate method for calculating the free energy of protein-ligand binding. However, before molecular dynamics can be used for accurate calculations, it is necessary to find a number of candidate molecules for the role of inhibitors. Such candidate inhibitors are usually found using docking followed by experimental testing. Docking programs are used for virtual screening of existing or virtual databases of ligands. Docking programs perform positioning of ligands by global optimization of the energy of protein-ligand complex [1]. Docking programs are highly demanded, but their accuracy is generally too low for reliable prediction of ligand inhibitory activity. One of the reasons for this is the use of classical potentials, a force field, to calculate the energy of intermolecular interactions. To increase the docking accuracy, a quasi-docking procedure was proposed [2], which reveals a broad spectrum of low energy minima of a proteinligand complex and searches for a global energy minimum by a semiempirical quantum-chemical method. In this procedure a broad spectrum of low energy minima is found using a force field. A set of 25 carefully selected protein-ligand complexes is used to compare quasi-docking accuracy of two semiempirical quantum-chemical methods, PM7 and PM6-D3H4X. These are advanced semiempirical methods that describe well both dispersion interactions and the formation of hydrogen and halogen bonds, which play an important role in the interaction of ligands with proteins. The quantum-chemical calculations are made using the MOPAC program, and influence of solvent is taken into account by the COSMO implicit solvent model. The spectra of low energy minima of protein-ligand complexes are found using the FLM supercomputer generalized docking program [3]. It is shown that PM7 method demonstrates better docking positioning accuracy and better correlation calculated and measured value of protein-ligand binding enthalpy.

The work was financially supported by the Russian Science Foundation,

Agreement no 21-71-20031.

\section{References}

[1] V. Sulimov, D. Kutov, A. Taschilova, I. Ilin, E. Tyrtyshnikov and A. Sulimov, Current Topics in Medicinal Chemistry, 2021, 21(6), 507-546.

[2] V. Sulimov, D. Kutov and A. Sulimov, Current Medicinal Chemistry, 2019, 26(42), 7555-7580.

[3] A. Sulimov, D. Kutov, I. Ilin and V. Sulimov. Biomeditsinskaya khimiya. 2019, 65(2), 80-85. 